\title{
Vaginal birth after Caesarean risk decision-making: Australian findings on the mothers' perspective
}

\author{
Pam McGrath B.Soc. Wk. MA PhD \\ Director and NHMRC Senior Research Fellow, International Program of Psycho-Social Health Research (IPP-SHR), \\ Central Queensland University, Brisbane, Queensland, Australia \\ Emma Phillips BA LLB (Hons) \\ Research Officer, International Program of Psycho-Social Health Research (IPP-SHR), Central Queensland University, Brisbane, Australia
}

\section{Grahame Vaughan MBBS MRCOG FRANZCOG}

Director (Retired), Department of Obstetrics and Gynaecology, Redland Hospital, Bayside Health District, Redlands, Queensland, Australia

Accepted for publication December 2009

McGrath P, Phillips E, Vaughan G. International Journal of Nursing Practice 2010; 16: 274-281 Vaginal birth after Caesarean risk decision-making: Australian findings on the mothers' perspective

The purpose of this paper is to explore, from the mothers' perspective, the decision-making experience with regard to subsequent birth choice for women who had previously delivered by Caesarean section. A qualitative methodological approach was taken to the exploration of mothers' knowledge of the risks of vaginal birth after Caesarean (VBAC) or elective Caesarean following a prior birth by Caesarean section. This paper presents the insights provided by the four women who chose VBAC. The health professionals' attitude to birth, and thus the support they offer to mothers, is predominantly pro-Caesarean. In view of the declining numbers of VBACs and the fact that the clinical literature documents risks for both elective Caesarean and VBAC, it is important for health professionals to be very sensitive and balanced in the information and support offered for the VBAC birth option.

Key words: mothers' perspective, qualitative research, risk, vaginal birth after Caesarean (VBAC).

\section{INTRODUCTION}

Although the literature suggests that few women want to deliver by Caesarean section (CS), ${ }^{1-4}$ Caesarean births now account for $23.3 \%$ of all births in Australia, ${ }^{5}$ $21.5 \%$ in the $\mathrm{UK}^{6}$ and $31.1 \%$ in the USA. ${ }^{7}$ Despite the

Correspondence: Pam McGrath, Centre for Social Science Research, School of Nursing and Health, Central Queensland University, Rockhampton, Queensland 4702, Australia, Email: pam_mcgrath@ bigpond.com endorsement by the American College of Obstetricians and Gynecologists supporting vaginal birth after Caesarean (VBAC) as a 'safe and acceptable option', and a National Institutes of Health recommendation that nonmedically indicated Caesareans should not be performed for pregnancies of $<39$ weeks of gestation and for women desiring several children, ${ }^{9}$ the repeat Caesarean rate in the USA is up to $92 \% .^{7}$

A decreasing number of women are attempting VBAC, despite ample evidence that the practice is predominantly safe for mother and baby, ${ }^{10}$ and associated with improved 
physical and emotional outcomes, and fewer long-term consequences. ${ }^{11}$ The actual rate of women undergoing trial of labour is much lower than the population of women at risk of complications from VBAC would suggest. Although VBAC success rates are reportedly $\approx 75 \%,{ }^{10,12}$ in the USA, only $8.5 \%$ of women with a previous CS undergo trial of labour. ${ }^{7}$ Why more women who are eligible for $\mathrm{VBAC}$ do not attempt it raises many questions.

Women prefer elective Caesarean (EC) or VBAC for a variety of reasons, including prior birth experiences, personal expectations of the birth process, family or social obligations and medical recommendations. ${ }^{2,4,13-15}$ Major influences for a woman to choose VBAC are a woman's sense of control in the decision-making process, physician encouragement for VBAC and delivery-type outcome advantages, both physical and emotional. ${ }^{16}$ Some studies report physician and midwife support for $\mathrm{VBAC} ;{ }^{17}$ others report conflicting evidence. ${ }^{11}$ A study of women intent on VBAC found that health provider support was not as important as the support of family and friends. ${ }^{11}$

Understanding the impact health practitioners have on a woman's birth preference is an important aspect of the EC vs. VBAC debate. Medical advice remains a key factor for many women in choices for childbirth. The amount and type of information doctors provide to pregnant women, and the physician's practice style ${ }^{18}$ can significantly impact upon the CS rate. The difficulty in going against medical opinion has been documented. ${ }^{11}$

Maternal anxiety, rather than consideration of risk, can be a deciding factor for many women. Appleton et al. found that parental anxiety, from the outset of pregnancy, was a key issue influencing whether a woman would attempt VBAC, therefore any hesitancy on the part of the health provider can be interpreted as reluctance by the woman. ${ }^{19}$ Women's fears and needs play a major part in the decision-making process; most perceive EC as a safer birth choice. ${ }^{4,11}$ In another study, women choosing VBAC had lower state anxiety and felt better prepared than women choosing repeat $\mathrm{CS} .{ }^{14}$

The risks of EC and VBAC cited in the medical literature and popular media have created confusion among health practitioners and mothers alike. Well-documented risks of CS include prolonged recovery, delayed breastfeeding, bleeding, infection and neonatal breathing difficulties. ${ }^{20}$ As the number of repeat Caesareans increases, so do the chance of reduced fertility, increased likelihood of abnormal placentation and surgical difficulties due to adhesions. ${ }^{21,22}$

Risks of VBAC also are heavily debated in the literature. Unsuccessful trial of labour necessitating emergency CS is a possibility, and although uncommon, lifethreatening complications such as uterine rupture and perinatal death might occur. ${ }^{21,23}$ The risk of uterine rupture is estimated at $0.5-1 \% ; ;^{23}$ however, this depends on several factors. Risk of uterine rupture with VBAC is increased $(1.8 \%)$ for two prior Caesareans. ${ }^{10}$ Also, the time interval between pregnancies affects the chance of uterine rupture: $2.3 \%$ in women with an interval $<18$ months since prior Caesarean, $0.9 \%$ in women with an interval $>2$ years. ${ }^{24,25}$ Induction of labour is possibly associated with an increased risk for uterine rupture. . $^{10,23,26}$ Importantly, prior vaginal delivery is protective against uterine rupture, with a success rate of $86.6 \%$ in this group. ${ }^{10,23}$

The perspective of pregnant women regarding birth risks following prior CS is not well understood. We conducted a qualitative study designed to explore, from the mothers' perspective, the decision-making experience with regard to subsequent birth choice for women who had previously delivered by CS. Only $20 \%$ of the mothers in the study chose VBAC. This article presents their understanding of the risks of $\mathrm{VBAC}$ and the extent to which this influenced their birth choice.

\section{METHODS The research}

The study was conducted by a senior research fellow at the Central Queensland University in association with a Director of Obstetrics and Gynaecology at Redlands Hospital, Queensland. The aim of the research was to explore from the mothers' perspective the process of decision-making about mode of delivery for a subsequent birth after a previous CS. The research project was initiated by the then Director who was keen to explore in-depth the subtleties of the mothers' experience with birth decision-making. The findings from the study are rich and dense and will be published separately as a number of articles. The findings presented in this article are from the data that describe the perspective of the mothers who chose VBAC on risks associated with VBAC and EC, and their experience with discussing such risks with the health professionals providing their obstetric care. 
Ethical consent to conduct the study was obtained from the university and hospital Human Research Ethics Committees. Participants were verbally informed of their rights in research and written consent was obtained for participation in the research.

\section{Methodology}

Descriptive phenomenology was chosen as the theoretical framework, as it underpins a research method that explores the 'lived experience' of people from the 'inside' perspective of the individuals involved in the experience. ${ }^{27}$ As Spiegelberg explains, descriptive phenomenology is:

the direct exploration, analysis, and description of particular phenomena, as free as possible from unexamined presuppositions, aiming at maximum intuitive presentation. ${ }^{28}$

In this case, the phenomenon is mothers' lived experience regarding decision-making about the mode of delivery for a subsequent birth following a CS. The discussion of decision-making extended to immediate birthing issues such as bonding and breastfeeding. As inductive, phenomenological, qualitative work, the reporting of findings is based on a commitment to the participants' point of view with the researcher playing the role of coparticipant in the discovery and understanding of what the realities are of the phenomena studied. ${ }^{29,30}$

\section{Participant group}

The findings presented in this article are from a study that interviewed 20 women who had all had a previous CS and had a subsequent birth at Redlands Hospital 6 weeks before the interviews, which were conducted in June 2008. The prospective participants were notified about the study by a letter from the hospital. The participants were consecutively enrolled from a list of mothers who gave birth during the specific month from the hospital records. The potential participants were approached by a university project officer independent of the hospital and given the written consent package along with a verbal description of the project. If agreeing to participate, a written consent was obtained before the commencement of the interviews. There were 20 mothers enrolled in the study. Of these 20 women, two gave birth by VBAC, two attempted VBAC and 16 chose EC. With the exception of two mothers who had three births (all of which were CS) and one mother who had four children (which included two natural births and two CSs), all the other mothers had two children with the previous birth being CS. All of the mothers who either achieved VBAC or attempted VBAC (TVBAC) had two children and their previous birth was CS.

Of the 20 mothers, $13(n=13)$ had an emergency CS as the prior birth and seven $(n=7)$ had EC. At the time of the interview, all of the women had two children with the exception of one mother who had four children and two mothers who had three children. All participants were either married or in a de facto relationship at the time of birth. Participants' mean age was 32 years, with an age range from 26 to 38 years old. All lived in the geographical catchment area of Redlands Hospital.

It is the subsection of findings from the women who either tried to or gave birth by VBAC that are presented here.

\section{Interviews}

Data collection was conducted through an iterative, phenomenological, qualitative research methodology using open-ended interviews conducted at the time and location of the participant's choice. The interviews were conducted by a psychosocial researcher employed by Central Queensland University and thus independent of the hospital. The interviews were informed by principles of 'phenomenological reflection' as outlined in the work of Van Manen. ${ }^{31}$ The line of questioning included the techniques of probing, paraphrasing or silence to explore the participant's experience. The interviews lasted for approximately an hour and were audio-recorded. The interviews were transcribed verbatim by a research assistant independent of the hospital.

\section{Analysis}

The language texts were then entered into the QSR NUD*IST (QSR International Pty Ltd, Doncaster, Victoria, Australia) computer program and analysed thematically. All of the participants' comments were coded into 'free nodes', which are category files that have not been pre-organized but are 'freely' created from the data. Thus, the data analysis is driven by all of the participants' insights, not by selected pre-assumptions of the coders. The research team did not mediate the findings but rather developed code titles that directly reflect the participants' statements ensuring the final analysis directly describes the phenomenon (birth decision-making) from the participants' perspective. The coding was established by an experienced qualitative researcher and completed by a team of research assistants who have extensive experience 
with coding qualitative data. There was complete agreement on the coding and emergent themes. The list of codes was then transported to a Word Computer Program (Word XP, Microsoft, Seattle, WA, USA) and organized under thematic headings. The findings presented in this article are from the data that describe the perspective of the mothers who chose VBAC on risks associated with the delivery modes of VBAC and EC, and their experience with discussing such risks with the health professionals who provide their obstetric care.

\section{Limitations}

The following findings are from four interviews with mothers who have either tried for or achieved a VBAC. Although small, this number is appropriate for phenomenological work, which seeks to document through openended methodology in-depth insights from smaller numbers of participants. Indeed, the most common sample size for this type of work includes between four and 40 participants. ${ }^{27}$ A small sample is considered sufficient when the researchers have chosen a group with an experience in common, as in this case mothers who were motivated to achieve a VBAC. As Holloway states, because of the depth of the research interviews and their analysis, the sample is generally very small. ${ }^{27}$ In phenomenological work, it is not the number of participants that is important but rather that participants are chosen mainly for their knowledge or an experience of a condition or event about which they can inform the researcher. ${ }^{32}$

There is another important reason, other than purely methodological, why the authors believe it is crucial to publish the insights from this group of mothers. The records from the Hospital Obstetric department echo the worldwide trend towards a decreasing number of women undergoing VBACs. ${ }^{11}$ Indeed, in this group of mothers, only two actually achieved a VBAC. The authors believe that the poignancy of these sharply declining numbers makes it an ethical imperative to make available through publication the insights of the small number of women who chose this mode of birth. This imperative is further strengthened by the fact there is very little literature available on the psychosocial aspects of the VBAC experience from the mothers' perspective. It is the authors' hope and expectation that the findings will make a contribution to the nursing literature by deepening our understanding of this critical but as yet unexplored birthing issue.

\section{RESULTS Facing the risks}

A significant factor at the core of the difficulty associated with decision-making in relation to attempting a VBAC is the notion of risk. The following findings describe the experience of mothers exploring the birth option of VBAC with their health professionals including the obstetricians at the hospital, the local general practitioner (GP) and the midwives. The VBAC mothers took personal responsibility for their birth delivery choice but were open to gaining information from health professionals, for example:

(TVBAC) I mean, they are there to give the advice and all the details that I needed to know. I just made my own decisions from all of that. I felt there were risks both ways.

Medical recommendations, especially during the birth, are experienced as powerful and difficult to go against. One of the highly motivated TVBAC mothers described being in labour for $14 \mathrm{~h}$, and just minutes before the delivery, when the head was visible, going to an emergency CS on the direction of her obstetrician.

\section{The hospital obstetricians}

The predominant perception of the message from the hospital by this group of mothers is that VBAC is seen as the most risky option:

(VBAC) There's almost a sense that a vaginal birth is more difficult and harder than a Caesarean. I would have thought it would have been the opposite. And, I mean, I agree that it's hard to obtain after you've had a Caesarean.

The mothers indicated that there were some doctors at the hospital supportive of VBAC but the problem is that it was not possible to remain exclusively under their care. As can be seen by the following, mothers had to see many different doctors with differing and inconsistent opinions:

(TVBAC) Every time you'd see a different doctor. I don't think I ever saw the same doctor. Some seem to be more towards the vaginal birth than the Caesarean and others the other way around. I think it's a bit of a personal preference really.

An example of a description of a supportive obstetrician is as follows: 
(TVBAC) Some of the doctors were supportive; they were really good. One particular doctor at the hospital talked towards the side of having a vaginal birth. She even said she had a vaginal birth after a 'caesar'. She was positive, I mean, she was good.

The following description of lack of support from the obstetrician suggests the focus on risk for the hospital:

(VBAC) I felt like they didn't want to be responsible. Yes, doesn't feel like you're supported at all.

The mothers reported that the risk from the doctors was couched in terms of possible death to the mother and child:

(VBAC) They just said that 'you have an increased risk', but they didn't say actually: 'there's no increased risk from the last birth that you had last year, last child'. They just said 'you have an increased risk of uterine rupture and uterine rupture basically means death of the child or mother, or both'. So that's what they said to me.

A key message highlighted by the hospital doctors is the risk for the mother of ending up in another emergency CS:

(TVBAC) But they also said to me . . that if I ended up with another emergency 'caesar', that again was a higher risk than having an elective 'caesar' because you were already in labour and all the rest of it. And they said, you'd need to look at that as well so you can make your decision.

Because of the risk, mothers were told they could not attempt VBAC if they were overdue:

(VBAC) Up at the hospital, yes it's possible, as long as you don't go overdue.

There is a clear distinction made between the mothers' acceptance of risk and the hospital's concern about risk. As the following example from a mother who read widely and came to the conclusion that VBAC was not the more risky option shows, the mother had to deal with not only her own personal risk but the sense of public risk from the hospital's perspective:

(VBAC) Well from the reading . . . No, I didn't think having previously given birth by Caesarean was an issue. But I guess from the moment I booked an appointment at the hospital I knew that that would be a complication for the hospital.

The general sense of risk for the hospital is seen as contributing to the lack of individual support for a VBAC that some mothers experience at the hospital:

(VBAC) And the risk factors ... I think a lot of the opposition I faced at the hospital had to do with risk, and . . like the more such risk to the hospital.

The hospital fear of risk is seen as the reason some doctors focus predominantly on the downside of VBAC:

(VBAC) You know, there might be one in a hundred chances that I have a uterine rupture, but they kept focusing on the fact that I might be that. Might be that one person that has it. Me, I was thinking, 'look I'm most likely going to be one of the 99'.

The problematic nature of the risk is highlighted throughout the encounter with the hospital as the following shows:

(VBAC) What they gave me was a sheet about the pros and cons of vaginal birth after Caesarean. And sort of like a disclaimer form saying that I understood what they were giving me. For the midwives . . the first midwife I saw said, 'look we're all for it but it is now a higher risk delivery'.

The notion that the hospital health professionals were more concerned about the risk than the mother was consistently voiced in the interviews, so the interviewer would provide the mothers with a summary of their concerns and allow them to comment. As the following shows, the mothers were clear about this idea:

(Interviewer summing up) So you read widely, and based on this reading you had a sense that you definitely wanted a vaginal delivery, and you felt very confident in that decision, but when you went up to the hospital you had a sense that they were more anxious about risk?

(VBAC) Yes.

Not the full story.

Some of the mothers reported that they were not given sufficient information on the risk from the hospital: 
(VBAC) So, there was not much information given. It was just this one sheet of paper and 'make sure you've read that and understand that and see you later'.

The predominant presentation of risk is viewed by this group of mothers as unbalanced because it favours an EC, for example:

(VBAC) I just feel like women aren't given the right information on both sides, all of the risks, we are not given the benefits of $V B A C$.

This group of mothers was aware of the risks but prepared to attempt a VBAC:

(VBAC) I was aware of the danger of tear of scar from previous Caesarean but prepared to take this risk.

The mothers accessed extensive information from elsewhere other than the hospital on risks associated with VBAC, such as:

(TVBAC) I was given a couple of lots of VBAC information that I had read through anyway in the books that I found. Plus my best friend got me some information downloaded off the net. In 'What to expect when you're expecting', there's a fair bit of information, as well.

Some mothers did their own search of the literature and came to a different conclusion about risk, which favours the VBAC option:

(VBAC) I had quite a few research studies that I had looked into about risk factors as well. Well funnily enough, I was looking at the statistics . . . because they always give you the risk factors of the uterine rupture: your number one risk factor. But I understood that my risk factor actually wasn't as high as I was led to feel...

The mothers looked at other risk factors, not just possibility of rupture, for example:

(TVBAC) But they couldn't find any sort of physical reason. I didn't have diabetes. I didn't have that sort of thing, so there was no reason really for me not to try a VBAC.

\section{General practitioner}

For both women who successfully delivered by VBAC, the support of their local GP was greatly appreciated:
(VBAC) Well I spoke with my GP first. My GP was quite passionate about having vaginal births as well, which was helpful. She gave me the name of a few books to have a look at.

(VBAC) GP was very supportive of my decision to give birth naturally.

However, for another mother who wanted a VBAC, there was a lack of support from the local GP who emphasized the ease of CS and the benefit of avoiding the stress of natural delivery:

(TVBAC) She's got four children herself and she's of the opinion... 'Look, if the pain gets too bad, you've already had a Caesarean. All the female gynaecologists themselves would just opt straight in and have a Caesarean. That's what they're all doing. . . Why go and put yourself through that again if you had such a terrible experience the first time? . . Look, I'm all for you wanting to try it. We'll give it a go, if it gets too hard and too bad, straight in for a Caesarean. You don't need to muck around.' That was her opinion. I said, 'Okay. I really want to do it though.'

\section{Midwives}

The support of midwives is noted as especially important. For some mothers, the midwives were supportive. However, there were also reports from others that the midwives placed too much emphasis on the risk and possible negative outcome, for example:

(VBAC) Every appointment that was after 40 weeks I felt bullied in a certain sense. I just came out of there feeling a bit battered. . . Told I needed to relax about it and go with the flow, that because of my determination I would be heartbroken. I mean the exact words of the fact that because I was so determined I wouldn't get what I wanted. I mean, as strong as I am, it took a lot of...

\section{DISCUSSION}

The findings indicate that for this group of women who either wanted or achieved a VBAC, there was evidence of a struggle for support for their birth choice from many of the health professionals involved in their care.

The VBAC mothers brought openness to information about risk, along with a determination to make up their own minds. They experienced the recommendation of doctors as powerful. The description of their experiences of the messages conveyed from the hospital obstetricians 
was perceived to be one of the overwhelming risks of VBAC and a preference for EC. Although there were obstetricians who were supportive of VBAC, and such support was appreciated, it was not possible for the mothers to engage solely with these doctors, because of the public health system. The mothers had different doctors on each visit and had to navigate a wide range of inconsistent attitudes and information. Fenwick and associates describe the pressure some women face from their obstetricians to have a CS. ${ }^{11}$ They argue that the inference that if something goes wrong with the attempted VBAC it will be the mother's fault is a difficult one to ignore, and many women find it hard, if not impossible, to stand by their own beliefs in the face of such pressure. ${ }^{11}$ The women in Fenwick et al.'s study were not anti-CS. They believed CSs have a place in modern childbirth, but they also wanted some control over the birthing experience, which they felt was lacking in their prior delivery by CS. Women who expressed a desire for control during delivery read widely about the risks of EC and VBAC and felt they had made an informed choice. ${ }^{11}$

The spectre of professional liability loomed large in our findings. There was a disparity between the mothers' understanding of risk, which was that there was risk associated with both modes of delivery, and that of the hospital, which viewed EC as safer. This general sense of the heightened risk of VBAC for the hospital was seen as interfering with the support for VBAC mothers' choice. One consequence from the mothers' perspective is that there was a lack of balanced discussion of the full positives of VBAC and the full negatives of EC. Women reported they were informed mostly of the risks of VBAC and the benefits of EC, raising the concern of what constitutes 'informed choice'. Some of the mothers did access information from a wide range of sources other than the hospital on the full range of risk factors, not just uterine rupture, and were prepared to accept what they considered to be the level of risk associated with a VBAC.

The local GP was also noted as a significant health professional in terms of support for birth delivery decision-making. Some, but not all, GPs offered full support for an attempted VBAC, which was appreciated by the mothers in their care. The midwives were also important health professionals caring for the women who similarly were divided in their support for or against the choice of VBAC.

In conclusion, birth is one of the important life events that have significance for all humans. The findings reported in this article explore an important but as yet underexplored topic associated with the birth - that is, decision-making as regards risk for a subsequent birth following a CS. The findings highlight that a range of disciplines, especially obstetrics, midwifery and general practice, impact on the birth decision. Women need to make informed choices in partnership with their healthcare providers, and feel supported in their birth choice. However, from the mothers' perspective, the extent of prenatal information provision was clearly biased towards EC.

\section{ACKNOWLEDGEMENT}

The study was funded by a Redlands Hospital/Central Queensland University Industry Grant.

\section{REFERENCES}

1 Gamble J, Health M, Creedy D. Women's request for a cesarean section: A critique of the literature. Birth 2000; 27: 256-263.

2 Hildingsson I, Radestad I, Rubertsson D et al. Few women wish to be delivered by caesarean section. BJOG: An International Journal of Obstetrics and Gynaecology 2002; 109: 618-623.

3 McCourt C, Weaver J, Statham $\mathrm{H}$ et al. Elective caesarean section and decision making: A critical review of the literature. Birth 2007; 34: 65-79.

4 Weaver J, Statham H, Richards M. Are there 'unnecessary' cesarean sections? Perceptions of women and obstetricians about cesarean sections for nonclinical indications. Birth 2007; 34: 32-41.

5 Australian Institute of Health and Welfare. Australia's Health. Canberra: AIHW, 20004. 2004.

6 Thomas J, Paranjothy S, RCOG Clinical Effectiveness Support Unit. The National Sentinel Caesarean Section Audit Report. London: RCOG Press, 2001.

7 Martin J, Hamilton B, Sutton P et al. Births: Final data for 2006. National Vital Statistics Reports: From the Centers for Disease Control and Prevention, National Center for Health Statistics. 2009; 57: 7.

8 American College of Obstetricians and Gynecologists (ACOG). ACOG Practice Bulletin: Vaginal birth after previous cesarean delivery. Clinical Management Guidelines. International Journal of Gynecological Obstetrics 1999; 66: $197-$ 204.

9 National Institutes of Health. State-of-the-science conference statement: Cesarean delivery on maternal request. Obstetrics and Gynecology 2006; 107: 1386-1397.

10 Macones G, Peipert J, Nelson D et al. Maternal complications with vaginal birth after cesarean delivery: A multicenter study. American Journal of Obstetrics and Gynecology 2005; 193: 1656-1662. 
11 Fenwick J, Gamble J, Hauck Y. Believing in birthChoosing VBAC: The childbirth expectations of a selfselected cohort of Australian women. Journal of Clinical Nursing 2007; 16: 1561-1570.

12 Landon M, Leindecker S, Spong C et al. The MFMU cesarean registry: Factors affecting the success of trial of labor after previous cesarean delivery. American Journal of Obstetric and Gynecology 2005; 193: 1016-1023.

13 Eden K, Hashima J, Osterweil P et al. Childbirth preferences after Cesarean birth: A review of the evidence. Birth 2004; 31: 49-60.

14 Gamble J, Health M, Creedy D. Women's preference for a cesarean section: Incidence and associated factors. Birth 2001; 28: 101-110.

15 Shorten A, Chamberlain M, Shorten B et al. Making choices for childbirth: Development and testing of a decision-aid for women who have experienced previous caesarean. Patient Education and Counseling 2004; 52: 307-313.

16 Ridley R, Davis P, Bright J et al. What influences a woman to choose vaginal birth after cesarean? Journal of Obstetrics, Gynecology and Neonatal Nursing 2002; 31: 665672.

17 Meddings F, MacVane Phipps F, Haith-Cooper M et al. Vaginal birth after caesarean section (VBAC): Exploring women's perceptions. Journal of Clinical Nursing 2007; 16: $160-167$.

18 Landon M. Vaginal birth after cesarean delivery. Clinics in Perinatology 2008; 5: 491-504.

19 Appleton B, Targett C, Rasmussen M et al. Knowledge and attitudes about vaginal birth after Caesarean section in Australian hospitals. Australian and New Zealand Journal of Obstetrics and Gynaecology 2000; 40: 195-199.

20 Declercq E, Sakala C, Corry M et al. Listening to Mothers II: The Second National U.S. Survey of Women's Childbearing Experiences. New York: Childbirth Connection, 2006. Available from URL:http://www.childbirthconnection.org/ listeningtomothers. Accessed 31 July 2008.
21 Dodd J, Crowther C, Huertas E et al. Planned elective repeat caesarean section versus lanned vaginal birth for women with a previous caesarean birth [Review]. Cochrane Database System Review 2004; 4.

22 Gilliam M. Cesarean delivery on request: Reproductive consequences. Seminars in Perinatology 2006; 30: 257-260.

23 Landon M, Hauth J, Leveno K et al. Maternal and perinatal outcomes associated with a trial of labor after prior cesarean delivery. New England Journal of Medicine 2004; 351: 25812589.

24 Bujold E, Gauthier R, Hamilton E. Maternal and perinatal outcomes associated with a trial of labor after prior cesarean delivery. Journal of Midwifery and Women's Health 2005; 50: 363-364.

25 Stamilio D, DeFranco E, Pare E et al. Short interpregnancy interval: Risk of uterine rupture and complications of vaginal birth after cesarean delivery. Obstetrics and Gynecology 2007; 110: 1075-1082.

26 Lydon-Rochelle M, Holt V, Easterling $\mathrm{T}$ et al. Risk of uterine rupture during labor among women with a prior cesarean delivery. New England Journal of Medicine 2001; 345: 3.

27 Holloway I. A-Z of Qualitative Research in Healthcare, 2nd edn. Oxford: Blackwell, 2008.

28 Spiegelberg H. Doing Phenomenology. The Hague: Nijhoff, 1975.

29 Sorrell J, Redmond G. Interviews in qualitative nursing research: Differing approaches for ethnographic and phenomenological studies. Journal of Advanced Nursing 1995; 21 : 1117-1122.

30 Streubert J, Carpenter D. Qualitative Research in Nursing: Advancing the Humanistic Imperative. New York: Lippincott, 1995.

31 Van Manen M. Researching Lived Experience. Albany, New York: State University of New York Press, 1990.

32 Sandelowski M. Sample size in qualitative research. Research in Nursing and Health 1995; 18: 179-183. 